\title{
Mit Afatinib auch tumorbedingte Beschwerden reduzieren
}

\begin{abstract}
Aktuelle Daten der LUX-Lung 3-Studie zeigen bei Patienten mit fortgeschrittenem nicht-kleinzelligem Lungenkarzinom (NSCLC) und positivem EGFR (Epidermal Growth Factor Receptor)-Mutationsstatus, dass der Tyrosinkinasehemmer Afatinib nicht nur die progressionsfreie Überlebenszeit signifikant verbessert, sondern auch tumorbedingte Beschwerden effektiver reduziert und besser kontrolliert als dies unter der Chemotherapie mit Pemetrexed/Cisplatin möglich ist. In der Konsequenz gaben die $\mathrm{Pa}$ tienten eine bessere Lebensqualität unter der Behandlung an.
\end{abstract}

Afatinib befindet sich derzeit in der klinischen Prüfung bei Patienten mit fortgeschrittenem NSCLC und positivem EGFRMutationsstatus.DerTyrosinkinasehemmer blockiert die für die Tumorpathogenese relevanten Signalwege, die über EGFR 1-4 aktiviert werden, irreversibel. In der randomisierten Phase-III-Studie LUX-Lung 3 wurde mit der Erstlinienbehandlung mit Afatinib fast eine Verdopplung der progressionsfreien Überlebenszeit der Patienten im Vergleich zur chemotherapeutischen Behandlung mit Pemetrexed/Cisplatin erreicht, einer der derzeit wirksamsten Erstlinientherapien für diese Patienten (Yang JC-H et al., J Clin Oncol 2012, 30 [Suppl]: LBA7500).

\section{Bessere Lebensqualität}

Die aktuellen Daten, die auf dem diesjährigen europäischen Krebskongress vorgestellt wurden, bestätigen die hohe Wirksamkeit der Substanz, erläuterte Prof. Dr. Vera Hirsh, Montreal/Kanada. Sie zeigen, dass sich auch tumorbedingte Beschwerden unter Afatinib signifikant reduzieren und deutlich länger stabil bleiben $(\mathrm{p}=0,007)$ als unter der Chemotherapie (Sequist L et al., ESMO 2012:P1229-PD) (Abb.). Die Patienten im Afatinib-Arm gaben deutlich weniger Husten $(p=0,244)$ sowie signifikant weniger Kurzatmigkeit $(\mathrm{p}=0,0103)$ und Schmerzen $(p=0,0513)$ an. Ermittelt wurde dies anhand validierter Fragebögen der European Organisation for Research and Treatment of Cancer (EORTC, QLQ-C30/ LC13).

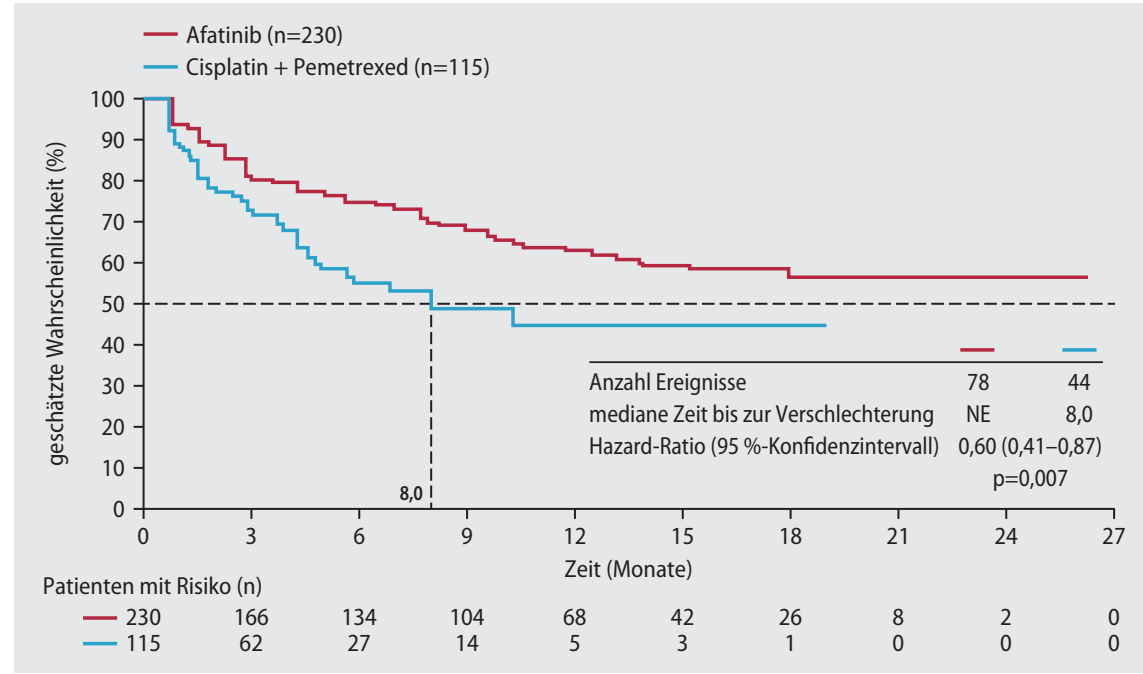

Reduktion tumorbedingter Beschwerden unter Afatinib (nach Sequist L et al., ESMO 2012: P1229-PD)

Bei der Befragung zur Lebensqualität wurden im Afatinib-Arm der LUX-Lung 3-Studie eine deutlich bessere Lebensqualität mit einem signifikant besseren globalen Gesundheitszustand $(\mathrm{p}=0,015)$ sowie deutlich bessere Funktionen im physischen $(p<0,001)$, emotionalen $(p=0,462)$, kognitiven $(p=0,007)$ und sozialen $(p=0,442)$ Be- reich ermittelt. Dies ist laut Hirsh auch Ausdruck der besseren Symptomkontrolle mittels Afatinib. Zudem wurde Afatinib von den Patienten in der Regel gut vertragen.

Pressekonferenz der Boehringer Ingelheim Pharma GmbH\& CO. KG

Akute myeloische Leukämie

\section{Immuntherapeutische Ansätze in der Erhaltungstherapie}

Die immuntherapeutisch wirksame Erhaltungstherapie aus Histamindihydrochlorid und Interleukin-2 (HDC/IL-2) konnte, wie in Studien gezeigt wurde, bei Patienten mit akuter myeloischer Leukämie (AML) in erster kompletter Remission, die nicht für eine allogene Stammzelltransplantation in Frage kamen, das Rezidivrisiko reduzieren und die Remission stabil erhalten (z.B. Brune M et al., Blood 2006, 108:89-96).

Experten zufolge nimmt die immuntherapeutische Erhaltungstherapie einen wichtigen Stellenwert ein, um dem Behandlungsziel - eine Langzeitremission
- näher zu kommen. In neueren Studien zeigte sich auch, wie wichtig die genaue Klassifikation der AML ist: Demzufolge führte HDC (Ceplene ${ }^{\circledast}$ )/IL-2 bei NichtM2-AML, insbesondere bei M4/M5Leukämien, zu besonders guten Resultaten, da diese Subtypen funktionelle Histaminrezeptoren exprimieren, die gezielt durch die Immuntherapie mit HDC/ IL-2 inhibiert werden (Aurelius J et al., Haematologica 2012, 97:1904-1908; Thorén $\mathrm{FB}$ et al., EHA 2011:\#056).

Die Behandlung mit HDC/IL-2 dauert 81 Wochen und erfolgt zyklisch.

nach Informationen der Meda Pharma GmbH \& Co. KG 